\title{
Perception at the blind spot and similarity grouping
}

\author{
N. KAWABATA \\ Electrotechnical Laboratory, Niiharigun, Ibaraki, Japan
}

\begin{abstract}
Perceptual completion at the blind spot was investigated by using patterns with a vertical blank stripe. The perceptual completion occurred across the vertical blank stripe in the test pattern, which was presented at the blind spot when a similarity grouping based on differences in the property of elements constituting the vertical blank stripe pattern was formed. There was no interaction between similarity groups in the filling-in process. The results obtained here indicate that perceptual completion is generated after similarity grouping, and that similarity groups are independent of each other in perceptual completion. The results seem to suggest that filling-in is closely related to a segregative process based on stimulus differences.
\end{abstract}

It is well known that a stimulation pattern that is displayed across the blind spot is completed in the visual information handling process and is perceived as a complete pattern. Information processing in perceptual completion requires that certain conditions be fulfilled, for example, the presence of a pair of stimuli facing each other across the blind spot (Kawabata, 1982). However, perceptual completion cannot be described by a linear summation of filling-in patterns, inferred from the local perceptual completion (Kawabata, 1983). Perceptual completion at the blind spot is affected by the global property of the stimulus presented around the blind spot: it is inhibited by the presence of a gap in the stimulus pattern (Kawabata, 1983; Le Grand, 1967). If the gap fails to inhibit completion, it means that certain information processing has been done before such an inhibitory process takes place.

It has been suggested that information processing, such as perceptual grouping, takes place before attention focuses (Beck, 1972). Similarity is one of the most important of the Gestalt principles in perceptual grouping. Similarity grouping is produced by the similarity of figural elements. Figural variables involved in producing a similarity grouping include slope of elements, element density, distance between elements, subjective brightness, etc. Therefore, when the property of the elements constituting a part of the pattern differs from that of the elements in the other part of the pattern, similarity grouping occurs. Then, it is expected that elements with a different property will be perceptually segregated from the other elements. This may affect the formation of edges and the gap in the pattern. If the completion is affected by the similarity grouping, it is expected that the completion will occur after the similarity grouping occurs. If generation of the completion depends on the

The author's mailing address is: Electrotechnical Laboratory, 1-1-4 Umezono, Sakuramura, Niiharigun, Ibaraki 305, Japan. similarity grouping, then completion at the blind spot will give us a measure for looking at perceptual grouping. On the other hand, if completion is not influenced by the similarity grouping, the property of the edges and gap inhibiting the completion would be more general than that of the perceptual grouping. In this paper, perceptual completion at the blind spot is studied from the viewpoint of similarity grouping, by changing such properties of figural components as orientation and density of the line segments.

\section{GENERAL METHOD}

Stimulation patterns were displayed on a graphic display (21-in. CRT with P31 phosphor). The subject sat in a darkened room, $50 \mathrm{~cm}$ from a CRT display, with his or her face fixed in a chinrest. The subject fixated, with right eye alone, a target presented on the CRT display. The subject triggered presentation of the test pattern while fixating the target. The test pattern composed of brilliant lines approximately $2 \mathrm{~min}$ wide, was displayed for $0.5 \mathrm{sec}$. The target was a spot $5.0 \mathrm{~min}$ in diameter. The luminance of the line was $8.2 \mathrm{~cd} / \mathrm{m}^{2}$, and that of the background, approximately $0.001 \mathrm{~cd} / \mathrm{m}^{2}$. The blind spot was mapped by using a flashing spot. In a run of trials, the order of test pattern presentation was randomized. Usually 30 measurements were made for one test pattern for each subject. Four subjects with normal or corrected-to-normal acuity participated in the experiments. Each experiment used three subjects. Two of the subjects were tested in all experiments.

The instructions in Experiment 1 were: "I will show you a series of test patterns. Each of the test patterns you will see is a striped rectangle traversed by a vertical blank stripe and a horizontal patterned stripe [the subject was shown the pattern]. The task I will ask of you is to judge whether the horizontal patterned stripe is seen as connected or separated. The connection of the horizontal patterned stripes may be blurred. If two areas of the horizontal patterned stripe are bridged by continuous blurring, it should be judged that the two areas are connected. A point, presented on the left side of the CRT, is the fixation point. Before each trial, you must fixate the point with the right eye alone, and when ready you can trigger the presentation of the test pattern by pressing the switch."

In order to familiarize the subject with the stimuli, the entire set was shown in the training session. The instructions in Experiments 2-4 were essentially the same as in Experiment 1, except 
that the examples presented were chosen from the patterns used in Experiments 2-4, respectively, and the subject was asked to judge whether the stripes, presented at a central horizontal zone in which the horizontal patterned stripes had been displayed in the previous experiment, were seen as connected or separated.

The width of the vertical blank stripe in the test pattern, that is, the gap length, used for these experiments was selected in such a way that the horizontal completion might occur stably when only the horizontal zone in the test pattern was presented around the blind spot.

\section{EXPERIMENT 1}

Perceptual completion occurs across the blind spot when a stimulation pattern is presented around it. However, a vertical blank stripe running through the stimulation pattern inhibits completion if the length and width of the vertical blank stripe are appropriate (Kawabata, 1983). Such a vertical blank stripe pattern may be bounded on each side by two rectangular areas striped either vertically or horizontally, as shown in Figure 1. If the vertical line length in this figure is shorter and under the vertical diameter of the blind spot, perceptual completion occurs across the blind spot.

Now, let us form another test pattern by inserting a central horizontal zone striped in an orthogonally different orientation from that in the bilateral rectangles, as shown in Figure 2, and present it so that the two stripes cross each other at the blind spot. In these patterns, vertical edges and a vertical gap between them may be formed globally. If the vertical edges and the gap inhibit perceptual completion across the blind spot horizontally, a vertical blank stripe should be perceived over a horizontal patterned stripe. On the other hand, if a horizontal patterned stripe crossing the vertical blank stripe is visible, it means that the proclivity of the vertical edges to inhibit completion is not operating and the horizontal completion occurs independently of the surrounding pattern.

\section{Method}

In this experiment, a series of test patterns was presented in which only the line spacings of the stripe in the horizontal
(A)

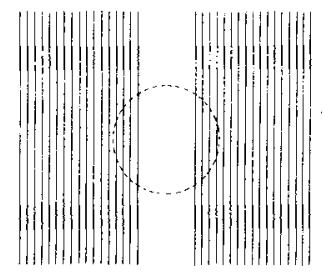

(B)

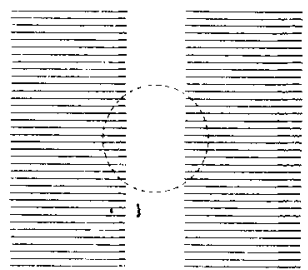

Figure 1. Examples of patterns made up of parallel lines with a vertical blank stripe. Pattern $A$ is composed of vertical parallel lines, and Pattern B of horizontal ones. Perceptual completion across the blind spot horizontally is deteriorated when the vertical blank stripe pattern is presented around the blind spot. A dotted line shows schematically the relative position of the blind spot when the pattern is presented.
(A)
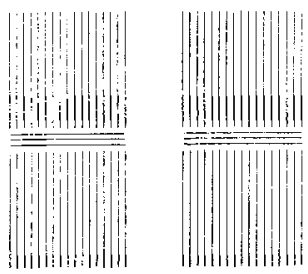

(B)

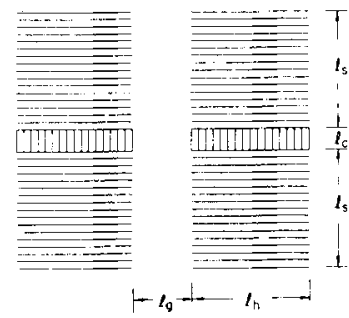

Figure 2. Test patterns with the orientation of parallel lines at a central horizontal zone orthogonal to ones in four rectangles. Vertical $\ell_{c}$ and $\ell_{s}$ were 1.29 and $6.88 \mathrm{deg}$, respectively. Horizontal length $\ell_{h}$ was $6.88 \mathrm{deg}$. Width $\ell_{g}$ of the vertical blank stripe was selected to allow a better fit to the individual blind spot. The range of $l_{g}$ used in the experiments was 3.44 . 5.16 deg. The interspace of the parallel lines was $0.43 \mathrm{deg}$.

zone were changed. The subjects were asked whether or not they saw a horizontal patterned stripe crossing the vertical blank stripe.

\section{Results and Discussion}

The experimental results are given in Figure 3. A horizontal zone, composed of parallel lines of different orientation from that in the surroundings, was perceived as connected without any relation to line spacing, although there were some individual differences among subjects. As a control, the striped patterns in the horizontal zone were presented at the same eccentricity as, but outside, the blind spot. In this condition, however, completion did not occur. Therefore, it can be said that the experimental results were due to completion at the blind spot. That is, the perceptual completion occurred across the blind spot horizontally and was not deteriorated by the vertical blank stripe in the test pattern. In other words, the vertical blank stripe shown in Figure 2 did not inhibit perceptual completion across it at the blind spot. This suggests that perception of the vertical edges and gap is not properly formed and that the horizontal filling-in occurred independently of the four rectangles in the stimulus.

\section{EXPERIMENT 2}

In Experiment 1, the orientation of parallel lines in the horizontal zone of the test pattern was orthogonally different from that in the four rectangles. It was thought that a difference in orientation of the parallel lines might make the perceptual completion possible. Therefore, test patterns with parallel lines in the horizontal zone were composed with the same orientations as those in the four rectangles but with different line spacings (Figure 4). If vertical edges and gap globally formed in such a test pattern simply inhibit the perceptual completion across them, the filling-in process would fail to make completion across the vertical blank stripe. If completion is perceived across the blind spot horizontally, it means either that vertical edges and gap are not properly 
(A)

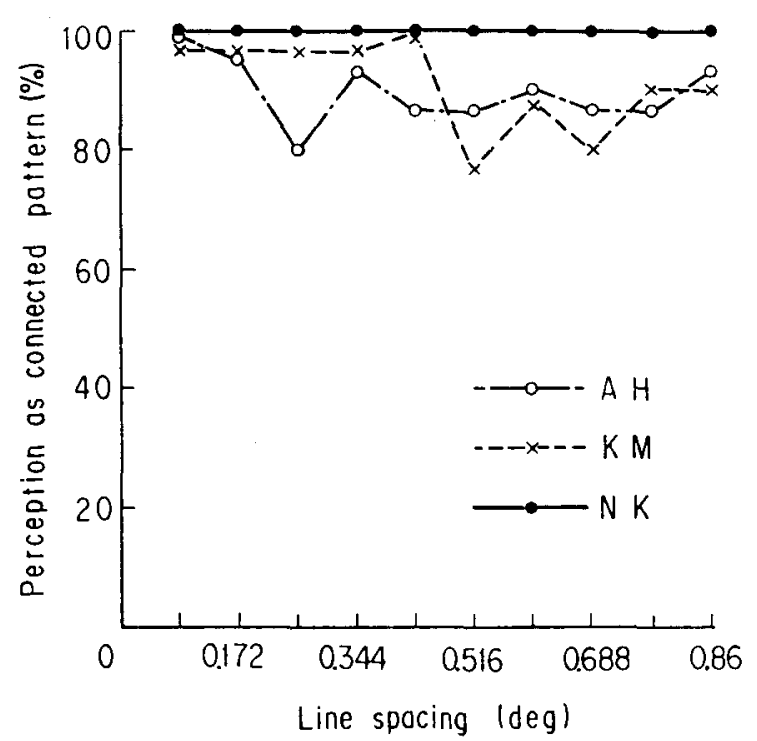

(B)

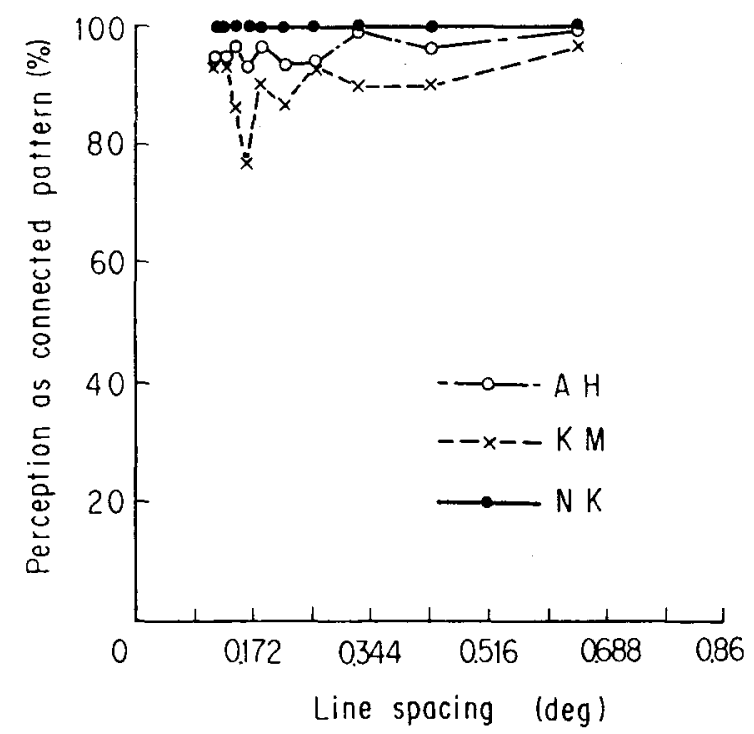

Figure 3. Perceptual completion across the blind spot horizontally for the test patterns shown in Figure 2. (A) gives the results for the pattern with the four rectangles made up of vertical parallel lines, and (B) gives those for the pattern with horizontal lines. The abscissa shows the line spacing of the parallel lines, and the ordinate denotes the probability of perception. The width of the vertical blank stripe was $5.16 \mathrm{deg}$ for Subjects A.H. and K.M.; it was 3.44 deg for Subject N.K.

formed or that the horizontal completion occurs independently of the vertical blank stripe.

\section{Method}

This experiment presented, in random order, a series of test patterns in which only line spacing in the horizontal zone was varied. Except for orientation, the line spacing in the four rectangles and the sizes of the horizontal zone and the four rectangles were the same as those of Figure 2.

\section{Results and Discussion}

The experimental results are shown in Figure 5. When the line spacing in the horizontal zone was similar to that in the four rectangles, the filling-in across the vertical blank stripe at the blind spot did not occur and a vertical gap was perceived shoving aside the horizontal patterned stripe, resulting in the deterioration of the

(A)
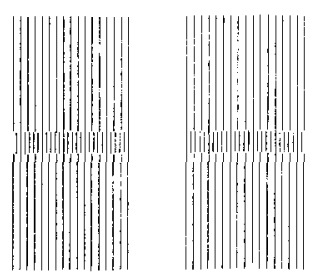

Figure 4. Test patterns in which the line spacing at the horizontal zone is different from that in the four rectangles. (A) is composed of vertical parallel lines, and (B) is composed of horizontal parallel lines. The sizes of the horizontal zone and of the four rectangles were the same as those in Figure 2. horizontal perceptual completion. When the line spacing in the horizontal zone differed from that in the four rectangles, a horizontal patterned stripe crossing the vertical blank stripe was perceived, that is, the horizontal filling-in was not deteriorated. With 0.43-deg line spacing in the four rectangles, the line spacing of 0.43 $\pm 0.043 \mathrm{deg}$ in the horizontal zone was regarded as identical and inhibited perceptual completion in the case of patterns composed of vertical parallel lines, although there were some individual differences among subjects. In case of horizontally oriented patterns, the range was $0.43 \pm 0.09 \mathrm{deg}$. It should be noted that there was no significant difference in perceptual completion between vertically and horizontally oriented test patterns, except for the tendency of a slightly broader range of line spacing in the latter case to inhibit perceptual completion $[t(4)=0.29, p>.7]$.

It is known that similarity grouping occurs without a complicated perceptual process. A difference in percepts at the blind spot due to the difference in line density may be based on the formation of similarity grouping. That is, when a test pattern is displayed, similarity grouping occurs first. Then one of the following occurs, depending on line spacing in the horizontal zone of the test pattern: (1) When the line spacing in the horizontal zone is similar to that in the four rectangles, the vertical blank stripe is formed and then the fillingin across it is inhibited. Thus, the horizontal perceptual completion is deteriorated. (2) When the line spacing in the horizontal zone is different from that in the four rectangles, the horizontal zone is segregated from the 
(A)

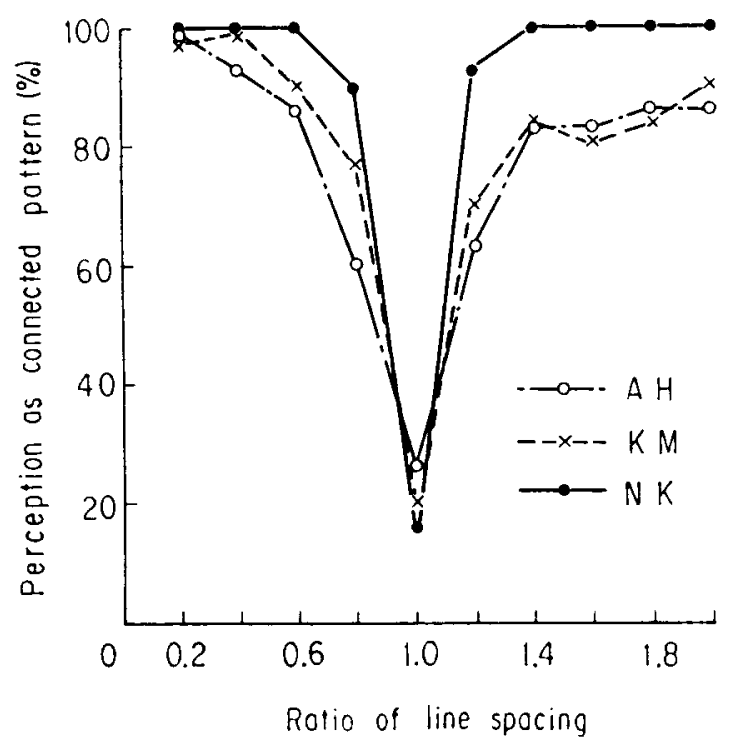

(B)

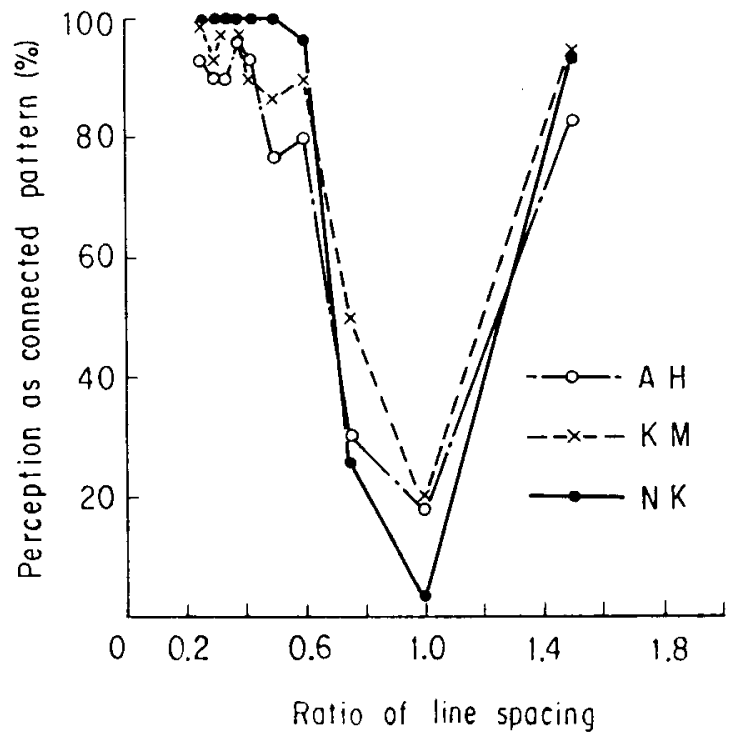

Figure 5. Perceptual completion across the blind spot horizontally for the test patterns shown in Figure 4. (A) gives the results for the pattern made up of vertical parallel lines, and (B) gives those for the pattern with horizontal lines. The abscissa shows the ratio of line spacings at the horizontal zone to those in the surrounding regions. When the line spacing at the horizontal zone was similar to that at the four rectangles, the completion was deteriorated.

four rectangles in the information handling process for the similarity grouping. Thus, the horizontal completion occurs across the vertical blank stripe at the blind spot independently of the four rectangles.

The difference in the line spacing that was effective enough to cause horizontal completion was smaller in the pattern composed of vertical parallel lines than in the horizontally oriented one. In the vertical pattern, remarkable subjective contours appear at a boundary between the horizontal zone and the four rectangles, since, because of the difference in the line spacing, the parallel lines in the horizontal zone and in the four rectangles are not aligned. Thus, the horizontal zone is easily separated from the four rectangles, and the parallel lines in the horizontal zone are grouped. On the other hand, in the pattern with horizontal parallel lines, the formation of the subjective contours is weaker at the boundary between the horizontal zone and the four rectangles, in contrast with the case of the vertical parallel lines, since there is no parallel-line termination array at the boundary. Thus, the formation of separated grouping may be difficult for the pattern in which the difference in the line spacings is less distinct.

\section{EXPERIMENT 3}

In the previous experiments, the line spacing and the orientation of the parallel lines at the horizontal zone of the test pattern constituted experimental variables in a series of test patterns, and the perceptual completion at the blind spot was investigated. Since the line spacing in the horizontal zone was different from that in the four rectangles in the test pattern, the horizontal zone and surrounding of the test pattern might have been separated into different groups on the bases of both the subjective contours at the boundaries and the difference in the line spacing. In order to study the effect of the subjective contours, the test patterns used included ones with parallel lines in the horizontal zone that had spacings and orientation that were the same as, but unaligned with, those in the surroundings.

\section{Method}

An example of the test patterns used is shown in Figure 6 . The instructions to the subjects were the same as in the previous experiments. In experiments that had used a series of test patterns that differed only in phases in alignment, the results had fluctuated extensively inasmuch as the subjects' responses were greatly affected by their perceptions of the pattern presented first. Therefore, the test patterns involving phase differences were randomly mixed with test patterns used in Experiments 1 and 2 . In experiments using this series of test patterns, anchor phenomena in the subjects' responses disappeared, and the responses could be regarded as independent of each other.

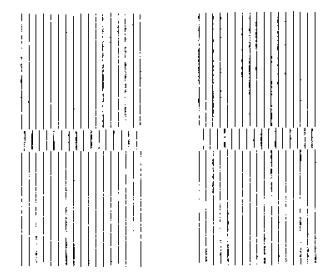

Figure 6. Test pattern with a 180-deg phase difference between the parallel lines at the horizontal zone and those in the four rectangles. The sizes of the horizontal zone and the four rectangles were the same as those of Figure 2. 


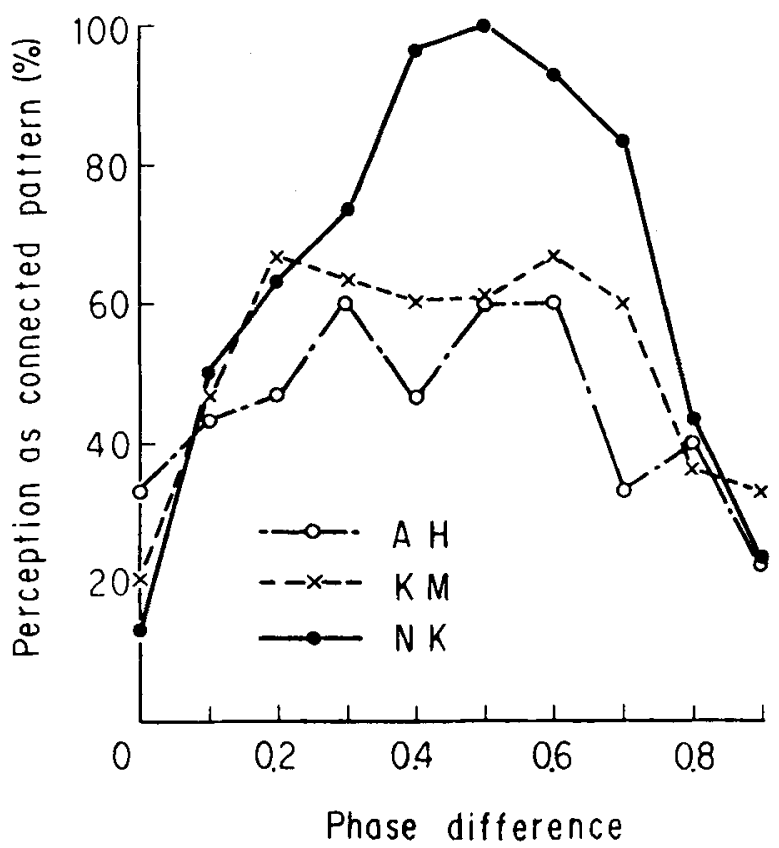

Figure 7. Perceptual completion across the blind spot horizontally for the test patterns with phase differences. Perceptual completion occurred across the blind spot for vertical blank stripe patterns with phase differences in parallel lines. The abscissa shows the deviation in phase of the parallel lines at the horizontal zone from those at the four rectangles. The value 0 indicates that both parallel lines are in phase, and the value 0.5 shows that both are completely out of phase. The ordinate indicates the probability of perception.

\section{Results and Discussion}

Experimental results obtained in this way are shown in Figure 7. Horizontal perceptual completion occurred across the vertical blank stripe at the blind spot for the patterns in which the phase of the parallel lines in the horizontal zone was different from that in the four rectangles, although the completion was not as strong as that in the previous cases. That is, the horizontal completion was not deteriorated by the vertical blank stripe shown in Figure 6. This indicates that the subjective contours based on the phase difference in the parallel lines separate them into different groups, and then the completion occurs independently in each group. This suggests that grouping may depend on the line spacing difference, but that the subjective contours also play a role in perceptual grouping.

\section{EXPERIMENT 4}

Is perceptual completion at the blind spot always inhibited by the presence of a blank stripe, even when the test pattern contains no local difference in the property of picture components? Let us consider the perceptual filling-in process by introducing a time lag in the presentation of a part of the test pattern.

\section{Method}

The test patterns used were ones in which, because of the vertical blank stripe, the horizontal completion did not occur across the blind spot. One of the test patterns, shown in Figure 8, with an excluded horizontal zone enclosed by broken lines was displayed first. After a random time-interval delay, the stripe at the horizontal zone was added, and the full pattern, including the excluded zone, was presented for $500 \mathrm{msec}$. If the completion is not affected by a time lag in the presentation, then the filling-in will not occur horizontally across the blind spot. If the time lag induces the completion, it means that the continuous vertical blank stripe is not perceived. It was expected that the perceptual completion might be inhibited at the end of the full-pattern display time in the second step, since the completion does not occur for the independent presentation of the full pattern. The completion may also be influenced by eye movements. Therefore, the judgment regarding the occurrence of completion should be made as quickly as possible after the second presentation. The instructions to the subjects were: "The first presentation of four rectangles will be followed after a certain time lag by a second one of full pattern. Your task is to judge whether the horizontal stripes are seen as connected or separated at the time they are displayed." The sizes of the horizontal zone and four rectangles were the same as those of the previous patterns. The line spacing in the test pattern was $0.43 \mathrm{deg}$. The display time for the initial exposure, that is, the time lag, was $0-180 \mathrm{msec}$.

\section{Results and Discussion}

Experimental results obtained under these experimental conditions are shown in Figure 9. The horizontal perceptual completion occurred across the vertical blank stripe at the blind spot for the time delay longer than $50 \mathrm{msec}$. For the time delay shorter than $30 \mathrm{msec}$, the completion did not occur. That is, two kinds of perceptual completion occurred, depending on the time delay between two presentations. For a shorter time delay, the pattern seems to be dealt with as if both parts were presented at the same time. Thus, the vertical edges and gap are formed at the blind spot, deteriorating the horizontal completion. For a time lag longer than $50 \mathrm{msec}$, the horizontal zone displayed later seems to be segregated as a group rather than incorporated into the four rectangles presented earlier. Then the horizontal
(A)

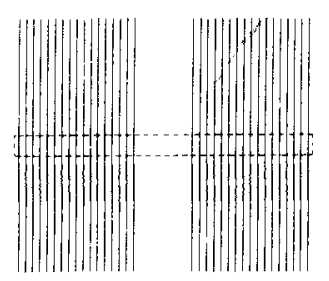

(B)

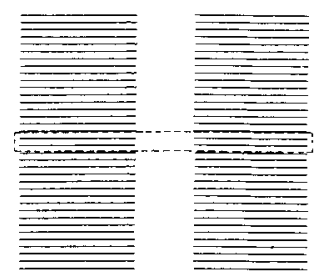

Figure 8. Test patterns used for Experiment 4. First, four rectangles, except for a horizontal zone enclosed by a dotted line, were displayed. After a random time-interval delay, the horizontal zone was added and both parts were presented for 500 msec. The sizes of the horizontal zone and the four rectangles were the same as those of the previous patterns. The dotted line is presented only for the convenience of explanation and did not appear in the test patterns used for the experiments. 
(A)

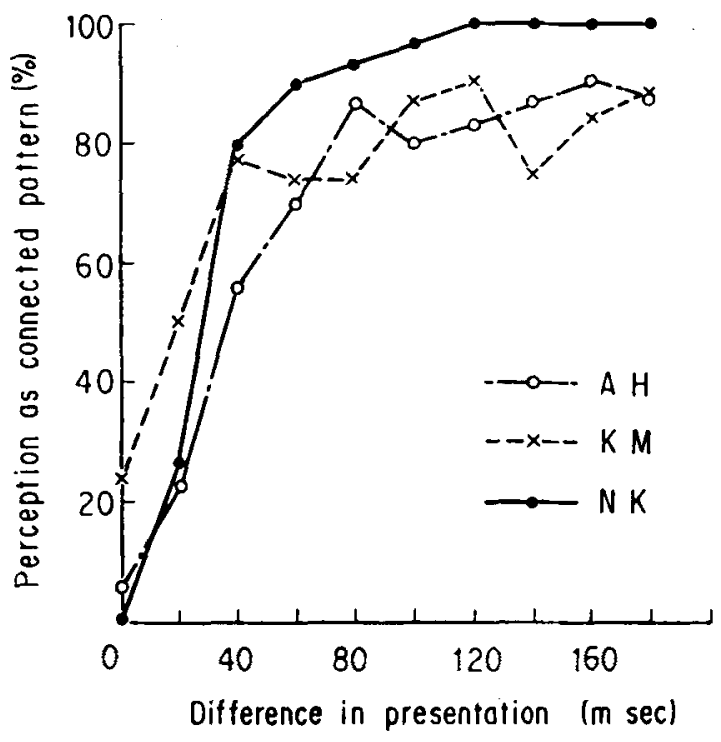

(B)

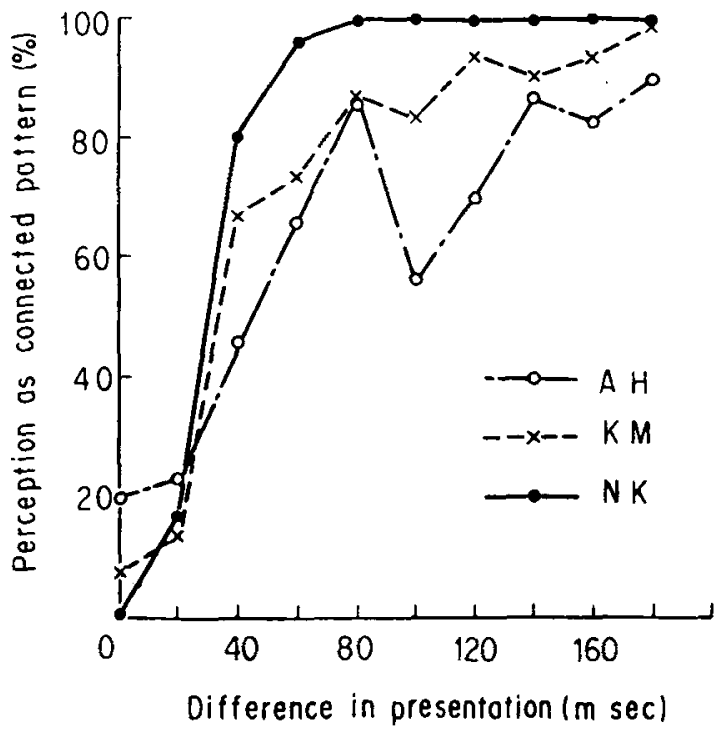

Figure 9. Perceptual completion across the blind spot horizontally for the test patterns shown in Figure 8. (A) and (B) give the results for test patterns $A$ and $B$ of Figure 8, respectively. The abscissa indicates the time lag in presentation of the horizontal zone after the four rectangles were displayed; the ordinate indicates the probability of perceptual completion.

zone is dealt with independently of the four rectangles in the filling-in process, and thus the horizontal perceptual completion occurs.

After the experiment, when asked about the criteria used in making their decisions, the subjects reported that they had responded on the basis of successiveness.

\section{EXPERIMENT 5}

The completion across the vertical blank stripe occurred depending on whether or not a similarity grouping was formed on the basis of the component property difference. To confirm this point of view additionally, the rate at which the horizontal patterned stripe was distinguished perceptually from the lines above and below it was measured by varying the horizontalstripe line spacings. If the rate of the occurrence of horizontal completion was similar to that of the perceptual discrimination, it would mean that the occurrence of perceptual completion can be predicted from the rating of the perceptual discrimination. On the other hand, the rating of the occurrence of completion provides a means for looking at perceptual grouping.

\section{Method}

The test patterns used for the present experiment were the same as those used in the previous experiments. The instructions to the subjects were the same as those in the previous experiments, except that they were asked to judge whether or not the stripe pattern in the horizontal zone was different from that in the surroundings.

\section{Results and Discussion}

For the test patterns shown in Figure 2, the stripe pattern in the horizontal zone could be distinguished clearly from that in the surroundings. Experimental results with the test patterns shown in Figure 4 are illustrated in Figure 10. The results obtained from the discrimination experiment are in agreement with those of the perceptual completion experiment as shown in Figure 5. Experimental results with the test patterns involving phase difference, as shown in Figure 6, are given in Figure 11. As can be seen in Figures 7 and 11 , the results of the two experiments indicate a similar tendency, although the high and low ratings are more clearly separated in the latter than in the former experiment. Grouping in the horizontal zone is weaker in the patterns involving a phase difference in the horizontal zone than in the patterns shown in Figure 4, since there is no difference in the line spacings between the horizontal zone and the surroundings. Therefore, the judgment in the discrimination experiment might be made based only on phase difference rather than grouping. On the other hand, the occurrence of completion depends on the grouping. Hence, the difference in ratings between them may be due to variation in judgment. The results obtained here, however, indicate that the occurrence of completion can be predicted from the rating in the discrimination; on the other hand, perceptual grouping can be investigated from the occurrence of completion.

\section{GENERAL DISCUSSION}

Perceptual completion does not occur across edges or a gap between them when a stimulation pattern that includes a blank stripe is displayed around the blind spot. The experimental results obtained here, however, indicate that perceptual completion does occur across 
(A)

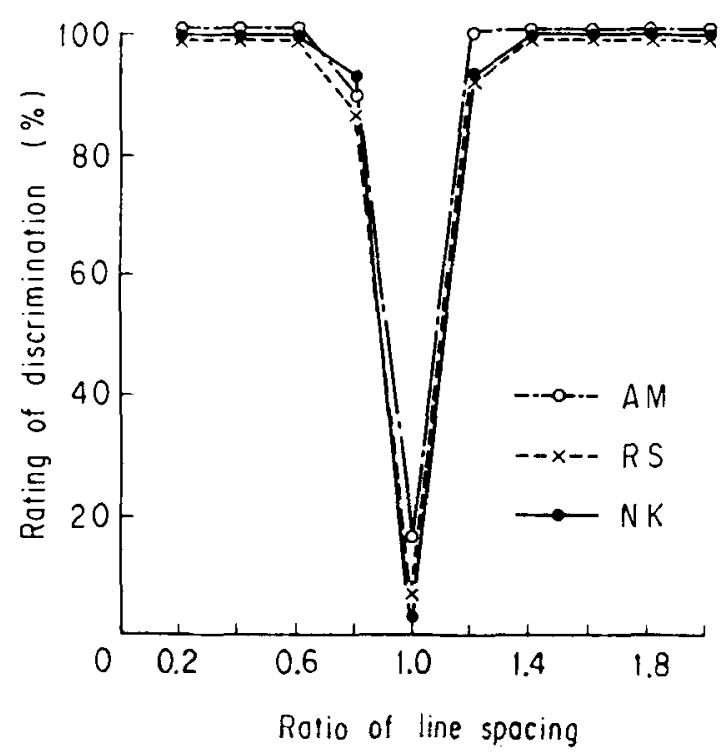

(B)

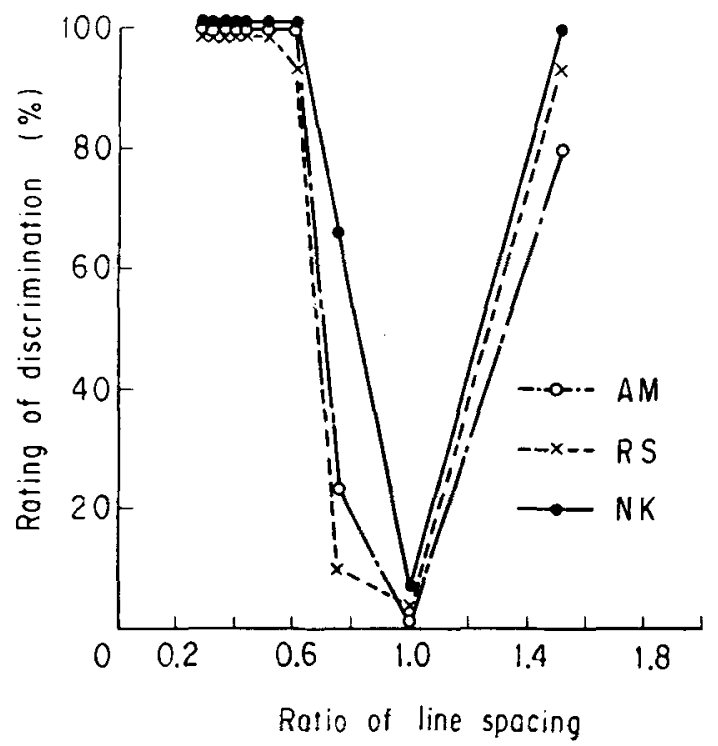

Figure 10. Rating of discrimination of the stripe at the horizontal zone from lines above and below in the test pattern shown in Figure 4. (A) gives the results for the pattern made up of vertical parallel lines; (B) gives those for the pattern with horizontal lines.

a vertical blank stripe when the property of the picture components bordering the blind spot differs from that of the remaining parts. In the experimental results, there were some deviations among subjects. These may be due to differences in judgment. In the judgment of completion, a blurred pattern crossing the vertical blank

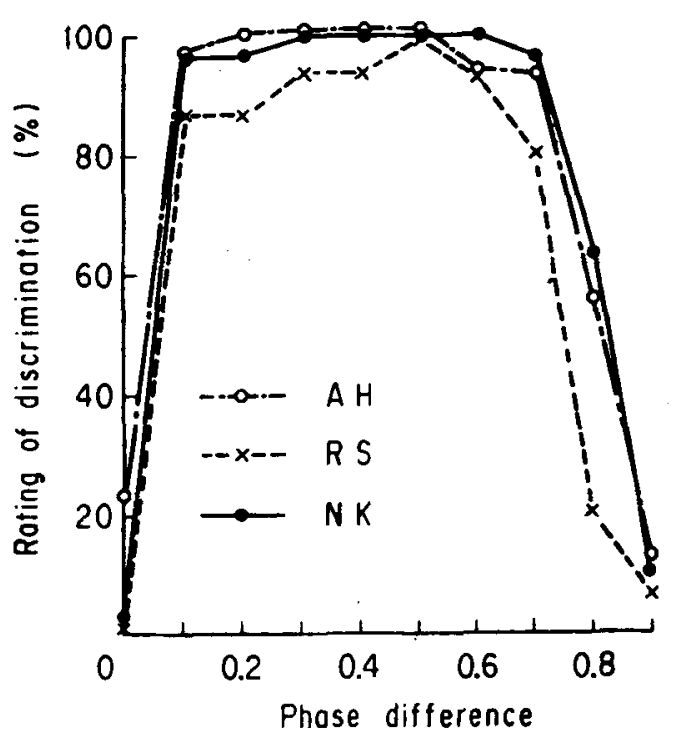

Figure 11. Rating of discrimination of the stripe at the horizontal zone from lines above and below in the test pattern shown in Figure 6. The abscissa shows the deviation of the parallel lines at the horizontal zone from the ones at the four rectangles. The value 0 indicates that both parallel lines are in phase, and the value 0.5 indicates that both are completely out of phase. stripe was allowed to be regarded as a connected pattern. Hence, it is possible that judgments may differ from individual to individual, depending on the degree of blurring. However, such deviations would not affect the significance of the results, since the range of variation in the completion was about $80 \%-100 \%$. Now, variations in fixation due to eye movements may be expected to affect experimental results. The effect of variations in fixation on the completion is of a suppressive nature, even if the eye moves during presentation of the stimulus. Therefore, the horizontal completion is not significantly affected by the eye movements, if any. In Experiment 2, when line spacings in the horizontal zone and four rectangles were sufficiently different, the horizontal completion occurred. Thus, variations in fixation based on eye movements do not affect the completion. When line spacings in two areas are similar, the horizontal completion do not occur. This may be due to variations in fixation during the experiments. As reported by Kawabata (1983), however, this is not due to eye movements. In that previous experiment, eye movements were checked by using a test pattern in which a portion to be displayed at the blind spot was deleted. That is, if the eye moves while the pattern is being presented, the stimulus pattern will not be perceptually completed and concavities at the edges of the test pattern will be perceived. In those experiments in which line spacings in the horizontal zone were identical to the four rectangles, two vertical edges were observed in the filled-in pattern. Therefore, it can be said that inhibitory effects on the completion reported here are not due to eye movements either. However, it is possible that eye movements might have depressed some estimates of the 
completion percentage. In a series of experiments in which the alignment of parallel lines and the onset of the presentation at the horizontal zone deviate from those of the four rectangles, the circumstances are the same. Therefore, it can be said that the perceptual completion reported here was not significantly affected by eye movements even if they occurred. In the test patterns used here, the blank completion was in the vertical direction and the pattern completion was in the horizontal one. This might have affected the results, because the blind area is approximately oval and its vertical diameter is somewhat longer than the horizontal diameter. However, the vertical and horizontal lengths of the test patterns were sufficiently longer than those of the blind area to decrease the effect due to a difference between the vertical and horizontal sizes of the blind area. Therefore, the completion would not be significantly affected by them. Now, the perceptual completion occurred across the vertical blank stripe when orientation or line spacing of parallel lines in the horizontal zone was changed or when a temporal difference in the presentation of a part of the stimulus pattern was introduced. These results suggest the existence of a process that detects differences in orientation and in line spacing of parallel lines before the filling-in process takes place. The completion occurs across the vertical blank stripe if such differences are detected in the pattern.

It has been known that similarity grouping is formed on the basis of the similarity of orientation and density of components, such as line segments constituting a figure (Olson \& Attneave, 1970). Such similarity grouping is thought to be formed on the basis of the response of the visual system to stimulus differences before attention focuses (Beck, 1972). In the test patterns used here, no doubt, information for the formation of similarity grouping would be processed in a manner that depended on the properties of the figural components. If similarity grouping is formed after the filling-in process, the perceptual completion must occur without any relation to the similarity grouping. The experimental results, however, indicate that the completion is greatly influenced by mixing components of different orientation or density in a part of the stimulus pattern. This suggests that the completion is remarkably affected by the similarity grouping. In other words, when a stimulation pattern is presented, the information for similarity grouping is processed on the basis of the similarity in components of the pattern, and subsequently the information for the perceptual completion is processed.

Now, the results also show that interaction between similarity groups, segregated on the basis of differences in the property of components, is absent or very weak in the filling-in process. That is, the perceptual completion depends on whether or not each group satisfies some conditions. This may suggest that both the vertical blank stripe and the horizontal patterned stripe are filled in across the blind spot when the horizontal zone with different components is segregated on the basis of similarity from the stimulus pattern containing the vertical blank stripe.

The results of Experiment 3 indicate that the subjective contours, based on the phase difference in alignment, also play a role in similarity grouping, although it is not so powerful. This supports the viewpoint that in the patterns shown in Figure 4, similarity grouping is formed both on the basis of a difference in line spacings between the horizontal zone and the four rectangles and on the basis of the subjective contours at the boundaries between them. Thus, the sharp tuning in the deterioration of completion observed in Figure 5A may also be explained by the differences in both line spacings and subjective contours.

In Experiment 4, the results show that the perceptual completion occurred across the vertical blank stripe if a part of the pattern was displayed with an appropriate delay. This suggests that similarity grouping is formed through temporal differences in the presentation of the test pattern.

Now, similarity grouping is a segregative process based on the response of the visual system to stimulus differences before attention focuses. The experimental results obtained here indicate that information processing for perceptual completion at the blind spot is done after such a segregative process takes place and is processed independently in each similarity group. This seems to suggest that the filling-in is closely related to the segregative process based on stimulus differences. It is pointed out that the segregative process may be a level of orientation analysis (Julesz, 1981). Thus, the present study suggests that the completion process must be at a level higher than the level of orientation analysis, since the perceptual completion occurs after similarity grouping. Cumming and Friend (1980) suggest that, on the basis of the magnitude of a tilt aftereffect, the fillingin process must be accomplished at a higher level in the visual system. The results here are in agreement with the results obtained from the tilt aftereffect.

\section{REFERENCES}

BECK, J. (1972). Similarity grouping and peripheral discriminability under uncertainty. American Journal of Psychology, 85, 1-19.

Cumming, G., \& Friend, H. (1980). Perception at the blind spot and tilt aftereffect. Perception, 9, 233-238.

Julesz, B. (1981). Textons, the elements of texture perception, and their interaction. Nature, 290, 91-97.

KaWABATA, N. (1982). Visual information processing at the blind spot. Perceptual and Motor Skills, 55, 95-104.

KaWABATA, N. (1983). Global interactions in perceptual completion at the blind spot. Vision Research, 23, 275-279.

Le Grand, Y. (1967). Form and space vision. Bloomington: Indiana University Press.

Olson, R. K., \& AtTneave, F. (1970). What variables produce similarity grouping? American Journal of Psychology, 83, 1-21.

(Manuscript received February 17, 1984; revision accepted for publication June 14, 1984.) 